AcCepted to ApJ LetTers

Preprint typeset using $\mathrm{IAT}_{\mathrm{E}} \mathrm{X}$ style emulateapj v. 03/07/07

\title{
DO BARYONS ALTER THE HALOS OF LOW SURFACE BRIGHTNESS GALAXIES?
}

\author{
Rachel Kuzio de Naray \& Kristine Spekkens \\ Department of Physics, Royal Military College of Canada, P.O. Box 17000, Station Forces, Kingston, ON, K7K 7B4, Canada \\ Accepted to ApJ Letters
}

\begin{abstract}
High-quality observations of dark matter-dominated low surface brightness (LSB) galaxies indicate that, in contrast to the triaxial, centrally-concentrated cuspy halos formed in collisionless simulations of halo assembly, these galaxies reside in round, roughly constant density cored halos. In order to reconcile these data with galaxy formation in the context of $\Lambda \mathrm{CDM}$, processes that alter the shape and density structure of the inner halo are required. We compile observational properties of LSB galaxies to evaluate the plausibility that a previously higher baryonic mass content and feedback from star formation can modify the dark matter halos of these galaxies. We also compare the properties of bulgeless disk galaxies formed in recent simulations to the LSB galaxy sample. We find that observational constraints on LSB galaxy star formation histories, structure, and kinematics make it difficult for baryonic physics to sphericalize and decrease the central density of the dark matter halos of LSB galaxies.
\end{abstract}

Subject headings: galaxies: evolution — galaxies: formation — galaxies: fundamental parameters

\section{INTRODUCTION}

There is now strong evidence that while galaxy formation is fundamentally driven by the hierarchical assembly of dark matter halos within the $\Lambda$ CDM framework (e.g., Navarro et al. 1996a; Stadel et al. 2009, and references therein), baryons play an important role in producing the observed structure of galaxies today. The incorporation of baryons in simulations of galaxy formation has been instrumental, for example, in producing realistic disk galaxies that are only moderately bulged (e.g., Brook et al. 2011; Guedes et al. 2011). Baryons have also been shown to be able to circularize the halo potential, transport angular momentum, and redistribute dark matter (e.g., Kazantzidis et al. 2010, hereafter K10; Hopkins et al. 2009; Weinberg \& Katz 2002, and references therein).

It is therefore plausible that baryons alter the cuspy ( $\rho \sim r^{-1}$ at small $r$ ), triaxial halos that are generically produced in collisionless simulations of halo assembly during galaxy formation. Baryons are thus a doubleedged sword: while the discrepancies between collisionless simulations and the observed properties of galaxies provide important constraints on the baryonic physics that shapes galaxies, this same physics prevents a direct comparison between the predictions of collisionless simulations and the structure of halos inferred from observations for the majority of galaxies.

There are classes of galaxies, however, for which the comparison between collisionless theory and observations is thought to be more direct. Conventional wisdom dictates that the halo structure of systems in which the dark matter dominates the mass density at all radii should resemble that produced by collisionless simulations. Low mass (dwarf) and low surface brightness (LSB) galaxies are attractive candidates for comparison to $\Lambda$ CDM predictions in this regard (e.g., de Blok \& McGaugh 1997).

Despite disputes over the interpretation of early kine-

Electronic address: kuzio@rmc.ca

Electronic address: Kristine.Spekkens@rmc.ca matic data, it is now clear that the halos inferred from observations of these systems are more core-like $\left(\rho \sim r^{0}\right.$ at small $r$ ) and rounder (at least in the regions probed by the data) than $\Lambda \mathrm{CDM}$ predicts, creating a long-standing conflict between theory and observation in these galaxies (see de Blok 2010, and references therein). Recently, however, simulations of dwarf galaxies by Governato et al. (2010, hereafter G10) have used feedback from star formation to remove low angular momentum gas during galaxy formation, producing a dark matter-dominated present-day system, preventing the formation of a bulge, and changing the initially cuspy dark matter halo into a more core-like halo. It therefore seems that baryons are likely integral to resolving the apparent discrepancy between dwarf galaxy properties and $\Lambda$ CDM predictions. Are baryons equally influential during the formation and evolution of LSBs?

The kinematics, structure, and star formation histories of LSBs are now well-characterized. In Table 1, we compile a subset of the properties of a representative sample of blue, late-type, bulgeless LSBs with central surface brightnesses fainter than $\mu_{0, B} \sim 23 \mathrm{mag} \operatorname{arcsec}^{-2}$. The table clearly illustrates that a) LSBs span a range of masses that crosses the typical dwarf/high surface brightness (HSB) galaxy threshold, b) they are strongly dark matter-dominated with most of their baryons in Hi, and c) they are metal-poor with low past and present-day star formation rates. It is important to note that LSBs are not rare; they comprise $\sim 50 \%$ of the general galaxy population (e.g., McGaugh et al. 1995).

Cosmological galaxy formation simulations that aim to produce LSBs with cored, spherical halos must be able to match the basic properties in Table 1. While the G10 simulations have utilized feedback to ease the conflict between collisionless halo predictions and dwarf galaxy observations, it remains unclear whether a similar mechanism applies to LSBs.

With the wealth of high-quality observations and improved galaxy formation simulations that are now available, the time is ripe to re-examine LSBs in the $\Lambda \mathrm{CDM}$ 
paradigm. In this Letter, we investigate the plausibility that currently favored baryonic processes (G10; K10) have altered the inner halos of LSBs in a way that reconciles the observed halo properties with collisionless simulation predictions. We focus on two key questions: 1) can baryons sphericalize LSB galaxy halos, and 2) can baryons turn LSB galaxy halo cusps into cores?

\section{SPHERICALIZING THE HALO}

K10 have shown that galaxies with massive disks can modify the shapes of their dark matter halo potentials. If the gravitational importance of the disk is significant enough, a triaxial halo can be sphericalized. Quantitatively, K10 found that the halo can be affected by the disk if the disk contributes at least $50 \%$ of the total rotation velocity at 2.2 times the disk scale length $\left(\eta \equiv V_{\text {disk }} / V_{\text {circ }} \gtrsim 0.5\right.$ at $\left.r=2.2 R_{\text {disk }}\right)$.

In Table 1, we list the total, HI, and stellar masses inside the last measured rotation curve point for the compiled LSBs, all of which have high-quality, twodimensional $\mathrm{H} \alpha$ and $\mathrm{HI}$ kinematics (see Table references). Also listed are the values of $\eta$ that we compute from the published mass models for each galaxy. By the K10 criterion, only 4 galaxies clearly have disks massive enough to sphericalize the halo; $\langle\eta\rangle \sim 0.41$ for the rest of the sample and there is no discernible trend with galaxy mass (see Figure 1a).

These subdominant LSB disks should therefore be embedded in triaxial dark matter halos. However, constraints on the elongation of the halo potential from the harmonic decomposition of high-resolution velocity field data (e.g., Trachternach et al. 2008) show the observations to be consistent with round potentials. Similarly, model velocity fields and rotation curves in asymmetric potentials are inconsistent with observations: kinematic signatures of asymmetric potentials (e.g., misaligned kinematic and photometric axes, twisted kinematic minor axis) without photometric counterparts are largely absent from high-resolution velocity field data (Kuzio de Naray et al. 2008, 2009; Kuzio de Naray \& Kaufmann 2011). The inner halos are round.

For comparison, we plot in Figure 1a the values of $\eta$ for three minimally-bulged disk galaxies formed in recent simulations. The G10 dwarf galaxy has just enough disk mass to sphericalize the dark matter halo. But the massive Brook et al. (2011) and Guedes et al. (2011) galaxies lie well above the sphericalization threshold, standing in stark contrast to LSBs of similar mass.

Present-day LSB disks are not massive enough to have reshaped their dark matter halos. In order to reconcile the observed round inner halos with collisionless triaxial halos using the K10 mechanism, LSBs must have had more baryons in the past; we return to this issue in Section 5.

\section{CHANGING A CUSP TO A CORE}

As discussed in Section 1, LSB galaxy rotation curves are often more consistent with cored inner halos than the cusps produced by collisionless simulations. In Table 1, we list the Alam et al. (2002) central halo density measure $\Delta_{V / 2}$ - the density within the radius at which the rotation curve falls to half its peak value, in units of the critical density — as a proxy for the inner halo profile slope in our LSB sample. We plot in Figure $1 \mathrm{~b}$ the re-
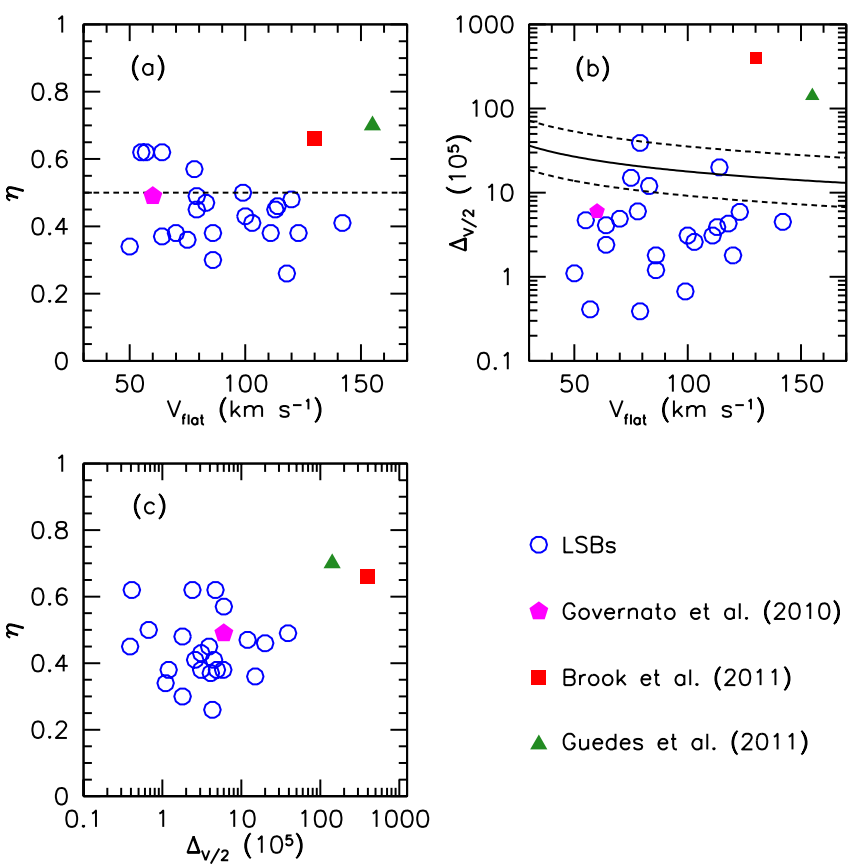

FIG. 1.- (a): Gravitational significance of the baryonic disk. Galaxies with $\eta>0.5$ (dashed line) are able to influence the shape of their dark matter halos. (b): The Alam et al. (2002) measure of central halo density. The lines indicate the expected relation and scatter for NFW halos and WMAP5 $\Lambda$ CDM parameters (Macció et al. 2008). (c): Gravitational significance of the disk versus halo central density. There is no correlation between these parameters for the LSBs.

sults for the LSBs and simulated galaxies, as well as the expected relation for NFW halos (Navarro et al. 1996a) in the WMAP5 $\Lambda$ CDM cosmology (Macció et al. 2008). For the LSBs, we find $\left\langle\Delta_{V / 2}\right\rangle \sim 6 \times 10^{5}$, approximately a factor of 4 lower than expected. To reconcile this number with $\Lambda$ CDM, LSBs must have been formed in cuspy halos that were altered by some physical process that produces no observable trends between $\Delta_{V / 2}$ and either $V_{\text {flat }}$ or $\eta$ (see Figure 1).

A number of ways of changing cuspy halos into cored halos have been suggested (e.g., dynamical friction, disk bars, supernova feedback), and generally invoke baryons. Feedback from star formation is of particular interest in the context of dark matter-dominated, bulgeless systems, since simulations suggest it can be effective at transforming cusps into cores (Navarro et al. 1996b; G10). Additionally, feedback in the G10 simulations produce $\Delta_{V / 2}$ and $\eta$ that are similar to LSB values. This feedback and outflow process can either be strong, impulsive and relatively violent, or as more recently suggested, can take place through a series of small(er) star formation events at $z>1$ (Pontzen \& Governato 2011, hereafter P11).

Invoking feedback to explain LSB galaxy halo cores therefore implies that the galaxies have had at least one period of early star formation and baryonic mass loss. This seems at odds with their low star formation rates, low surface densities and blue colors (see Table 1). We explore possibilities for reconciling LSB galaxy properties with the feedback mechanism for producing cores in Section 5.

4. UNDERSTANDING LSB PROPERTIES: THE ROLE OF MASS SURFACE DENSITY 
Low mass surface densities are likely key to many of the observed properties of LSBs. Star formation is both minimal and inefficient when the gas density is low. A galaxy that forms few(er) stars naturally has low metallicity, low surface brightness, a large gas mass fraction, and a large mass-to-light ratio, all properties describing the systems in Table 1.

One way to achieve low gas density in LSBs is to form them in underdense regions (e.g., Rosenbaum et al. 2009). External processes like tidal interactions and mergers that increase the gas density are minimized if the overdensities that eventually become LSBs were originally located in or near large-scale voids. This is an attractive formation scenario for LSBs as it would also satisfy the observational constraints that the galaxies are relatively isolated and located on the edges of large scale structure (Bothun et al. 1997; Rosenbaum et al. 2009).

The gas densities may also be kept low if LSBs form within high spin parameter halos (e.g., Dalcanton et al. 1997; Boissier et al. 2003). High angular momentum suppresses the collapse of the disk, leading to a galaxy with a larger disk size, lower surface brightness, and lower gas surface density than a low-spin galaxy of the same mass. Additionally, these galaxies will have larger dynamical mass-to-light ratios and rotation curves that are determined by the dark matter rather than the baryons. All of these characteristics apply to LSBs (e.g. Zwaan et al. 1995).

The challenge for LSB galaxy formation within $\Lambda$ CDM is to alter the halos, presumably via a baryonic process, in a way that preserves or produces the low present-day surface densities that explain the properties in Table 1 and Figure 1.

\section{RECONCILING LSB HALOS WITH $\Lambda$ CDM PREDICTIONS}

The work of K10 has demonstrated that massive baryonic disks can sphericalize inner halos, and that of G10 and P11 suggest that star formation feedback can flatten an inner density cusp in dwarf galaxies. Extending these ideas to the LSB regime, it may be possible to understand present-day LSB galaxy halo properties in the $\Lambda \mathrm{CDM}$ context if these systems had substantially more baryons in the past that were then blown out after star formation.

In the G10 simulations, ejecting "a few times the current stellar mass" is sufficient to reproduce observed dwarf galaxy properties after the halo core is formed. For the LSBs in Table 1, this conservatively translates to a blown-out baryonic mass of $\sim 6 \times 10^{9} M_{\odot}$. The value of $\eta$ before the mass loss is presumably higher as well, easing the tension between the present-day roundness of the inner halos and the K10 criterion.

Here, we discuss the plausibility of this model for round, cored halos in LSBs given their observed properties in Table 1. Specifically, we address how star formation would be induced (Section 5.1), the effectiveness of the subsequent feedback (Section 5.2), and whether early and/or multiple bursts of star formation are consistent with the star formation histories of LSBs (Section 5.3).

\subsection{Inducing Star Formation}

Mergers and interactions are effective ways to generate star formation by funneling gas to the galaxy center. This may be a tall order for LSBs considering that they have fewer, and more distant, neighbors than HSBs (Bothun et al. 1997; Rosenbaum et al. 2009). This mechanism is even more problematic if LSBs form in underdense regions and then migrate to their current locations (see Section 4). Observations support a quiescent evolutionary picture in which LSBs have had fewer major mergers and tidal interactions than HSBs.

Secular processes like bar and bulge formation are also effective at directing low angular momentum gas to the centers of galaxies where star formation can occur. These structures are largely absent from LSBs, however (Bothun et al. 1997); this is not surprising given that light disks are stable against bar formation (e.g., Mihos et al. 1997).

There is an additional hurdle to initiating star formation in LSBs if they form in high-spin halos (see Section 4). If this is the case, then LSBs are already at a disadvantage: there would be an even higher angular momentum threshold to overcome.

Initiating starbursts in LSBs therefore seems difficult. Nevertheless, if one manages to induce efficient star formation at some point in their evolution, how effective is the resulting feedback?

\subsection{The Effectiveness of Feedback}

The values of $\eta$ in Table 1 suggest that baryons must be removed from LSBs after their halos are sphericalized. For the lower mass LSBs, this may not be problematic. The G10 simulations have shown that star formation feedback can eject significant amounts of baryons from dwarf galaxies, a process that is in fact required to match observations (see also P11).

However, LSBs are not exclusively low mass (see Table 1), and supernova winds are not expected to be effective at ejecting baryons from systems with $V_{\text {flat }} \gtrsim 100$ $\mathrm{km} \mathrm{s}^{-1}$ (e.g., Dekel \& Silk 1986). Similarly, as discussed by Kereš et al. (2009) and references therein, unless the star formation rate is very high (as at high redshift), feedback and outflows do not eject large amounts of baryons in galaxies with $M_{\text {galaxy }}>10^{9} M_{\odot}$. This is the realm in which the LSBs we are considering fall; their gas masses alone are a few $\times 10^{9} M_{\odot}$ (see Table 1$)$. Brook et al. (2011) have shown that baryons are only temporarily blown out of high mass galaxies and eventually fall back to the galaxy disk and form stars. This scenario would be inconsistent with the $\eta$ values determined for higher mass LSBs today.

For a feedback scenario to be successful in LSBs, it must be effective at removing baryons from LSBs with a range of masses. Notwithstanding this challenge, if one assumes that enough star formation and feedback occur to alter LSB galaxy halos and blow out baryons, is the resulting stellar population consistent with the observed characteristics of LSBs today?

\subsection{LSB Galaxy Star Formation Histories}

Observations of the stellar populations, star formation histories, colors, and metallicities of LSBs place further constraints on the likelihood that baryons alter the dark matter halos and are subsequently ejected by star formation and feedback.

Attempts to constrain the ages of LSBs find stellar populations in the range of $1.5-6$ Gyr (Vorobyov et al. 
2009, and references therein), suggesting that the major star formation event took place at a redshift between $z \sim 0.2-0.4$ (Haberzettl et al. 2008). Substantial populations of old stars, which would exist if large-scale star formation had taken place in the past, are not observed; young stars, not a dominant population of old, low mass stars, are responsible for the blue colors of LSBs (see Table 1). LSBs are not the faded remnants of once-HSBs that have ceased star formation (e.g., Wyder et al. 2009).

LSBs are also metal-poor (see Table 1), indicating that they form relatively few stars over a Hubble time. Additionally, there is typically not much dust, a byproduct of star formation, in LSBs (see the discussion and references in Wyder et al. 2009).

Current star formation rates in LSBs are at least an order of magnitude lower than in HSBs (van den Hoek et al. 2000, see also Table 1), and the star formation efficiencies are only a few percent (e.g., Wyder et al. 2009). Observations and modeling indicate that the properties of LSBs are in good agreement with exponentially decreasing star formation rates combined with sporadic smallamplitude star formation events (e.g. van den Hoek et al. 2000; Vorobyov et al. 2009).

Given the observational constraints of gas-richness, low gas surface density, low star formation rates, and low abundances, the amount of past star formation in these galaxies could not have been very large; this is borne out by estimates of their past star formation rates (see Table 1). It is possible that LSBs exhibited episodic star formation and feedback at high redshift $(z \sim 2-4)$ that could alter their halos as suggested by P11, but that would not leave a detectable signature in their stellar populations. However, one still needs a mechanism to "shut off" this activity and replenish the pristine unevolved gas that is unique to LSBs.

\section{SUMMARY AND OUTLOOK}

Detailed spectroscopic and photometric observations of LSBs indicate that their inner dark matter halos are round and cored. In order to reconcile these properties with the triaxial, cuspy halos produced in collisionless simulations of halo assembly, mechanisms that sphericalize and decrease the central density of the halo must operate.

In this Letter, we have compiled observed kinematics, structure, and star formation histories of a representative sample of late-type, bulgeless LSBs to examine the feasibility of baryons altering their halos. Invoking the currently favored methods for sphericalizing and erasing cusps in dwarf galaxies implies that LSBs must have had more baryons in the past that were then blown out by feedback from efficient star formation. We have argued that this is an unlikely scenario given the well-established observational properties of LSBs.

The properties of our LSB sample and state-of-the-art simulated "bulgeless" galaxies are illustrated in Figure 1. The dwarf galaxy of G10 is similar to the observed LSBs of comparable mass, suggesting that if star formation is initiated, feedback and baryon removal can been effective at changing both the shape and density structure of the dark matter halo. There are stark differences at high mass, however, between the LSBs and simulated galaxies. Thus, while advances in simulating realistic galaxies have been made, no simulations yet resemble LSBs (nor claim to). Because the simulated massive galaxies retain or re-accrete the baryons ejected during star formation feedback, their central halo densities are higher than both the densities of LSBs of similar mass and expectations for $\Lambda \mathrm{CDM}$. This is most likely due to adiabatic contraction during the formation of the simulated galaxies.

What may be necessary for simulating LSBs with a wide range of masses is the combination of high(er) gas densities and low star formation efficiencies. Of the most recent simulations, G10 impose the highest gas density required for star formation, $n=100$ atoms $\mathrm{cm}^{-3}$, but this is just at the lower limit of the densities of giant molecular clouds. Restricting star formation to only the highest density regions (which are also physically small) would be consistent with very little molecular gas being detected in LSBs (e.g. Das et al. 2006), as the filling factor of these high density clouds would be low in a single resolution element of the observations. But because regions with high gas densities are physically smaller than regions with low gas densities, increasing the density threshold for star formation requires very high numerical resolution, making this a computationally expensive endeavor.

We reiterate here that while it is tempting to sweep LSBs into a "special" class of galaxies that form in a biased subset of halos (e.g. Macciò et al. 2007), LSBs are too numerous to be the tail-end of the galaxy distribution (e.g., McGaugh et al. 1995). Until numerical simulations can resolve the scales on which star formation is taking place and can implement a complete treatment of ISM physics allowing the properties of LSBs to be reproduced, these galaxies remain a problem for the $\Lambda \mathrm{CDM}$ picture.

We thank Fabio Governato and Stacy McGaugh for helpful comments on an early version of this manuscript, and acknowledge funding from the Natural Sciences and Engineering Research Council of Canada (NSERC).

\section{REFERENCES}

Alam, S.M.K., Bullock, J.S., \& Weinberg, D.H. 2002, ApJ, 572, 34 Bell, E.F. et al. 2000, MNRAS, 312, 470 (B00)

Boissier, S., Monnier Ragaigne, D., van Driel, W., Balkowski, C., \& Prantzos, N. 2003, Ap\&SS, 284, 913

Bothun, G., Impey, C., \& McGaugh, S. 1997, PASP, 109, 745

Brook, C.B., Stinson, G., Gibson, B.K., Roškar, R., Wadsley, J., \& Quinn, T. 2011, arXiv:1105.2562, submitted to MNRAS

Dalcanton, J.J., Spergel, D.N., \& Summers, F.J. 1997, ApJ, 482, 659

Das, M., ONeil, K., Vogel, S. N., \& McGaugh, S. 2006, ApJ, 651, 853

de Blok, W.J.G. 2010, Advances in Astronomy, 2010 de Blok, W.J.G. \& Bosma, A. 2002, A\&A, 385, 816 (D02) de Blok, W.J.G. \& McGaugh, S.S. 1997, MNRAS, 290, 533 de Blok, W.J.G. \& McGaugh, S.S. 1998, ApJ, 508, 132 (D98) de Blok, W.J.G., McGaugh, S.S., \& Rubin, V.C. 2001, AJ, 122, 2396 (D01)

de Blok, W.J.G., McGaugh, S.S., \& van der Hulst, J.M. 1996, MNRAS, 283, 18 (D96)

Dekel, A. \& Silk, J. 1986, ApJ, 303, 39

Governato, F. et al. 2010, Nature, 463, 203 (G10)

Guedes, J., Callegari, S., Madau, P., \& Mayer, L. 2011, arXiv:1103.6030, submitted to ApJ 
Haberzettl, L., Bomans, D.J., \& Dettmar, R.-J. 2008, IAUS, 244, 354

Hopkins, P.F., Cox, T.J., Younger, J.D., \& Hernquist, L. 2009, ApJ, 691, 1168

Hunter, D.A. \& Elmegreen, B.G. 2005, AJ, 128, 2170 (H05)

Hunter, D.A. \& Hoffman, L. 1999, AJ, 117, 2789 (H99)

Kazantzidis, S., Abadi, M.G., \& Navarro, J.F. 2010, ApJ, 720, L62 (K10)

Kereš, D., Katz, N., Davé, R., Fardal, M., \& Weinberg, D.H. 2009, MNRAS, 396, 2332

Kim, J.H. 2007, PhD thesis, University of Maryland (K07)

Kuzio de Naray, R., McGaugh, S.S., \& de Blok, W.J.G. 2004, MNRAS, 355, 887 (K04)

Kuzio de Naray, R., McGaugh, S.S., \& de Blok, W.J.G. 2008, ApJ, $676,920(\mathrm{~K} 08)$

Kuzio de Naray, R., McGaugh, S.S., \& Mihos, J.C. 2009, ApJ, 692, 1321

Kuzio de Naray, R. \& Kaufmann, T. 2011, MNRAS, 414, 3617

Liang, Y.C., Hu, J.Y., Liu, F.S., \& Liu, Z.T. 2007, AJ, 134, 759 (L07)

Macciò, A.V., Dutton, A.A., van den Bosch, F.C. 2008, MNRAS, 391, 1940

Macciò, A.V., Dutton, A.A., van den Bosch, F.C., Moore, B., Potter, D., \& Stadel, J. 2007, MNRAS, 378, 55

McGaugh, S.S. 2005, ApJ, 632, 859 (M05)

McGaugh, S.S., Bothun, G.D., \& Schombert, J.M. 1995, AJ, 110, 573

Mihos, J.C., McGaugh, S.S., \& de Blok, W.J.G. 1997, ApJ, 477, L79
Navarro, J.F., Frenk, C.S., \& White, S.D.M. 1996a, ApJ, 462, 563

Navarro, J.F., Eke, V.R., Frenk, C.S. 1996b, MNRAS, 283, 72

Pilyugin, L.S. \& Thuan, T.X. 2007, ApJ, 669, 299 (P07)

Pilyugin, L.S., Vílchez, J.M., \& Contini, T. 2004, A\&A, 425, 849 (P04)

Pontzen, A. \& Governato, F. 2011, arXiv:1106.0499, submitted to $\operatorname{ApJ}(\mathrm{P} 11)$

Rosenbaum, S.D., Krusch, E., Bomans, D.J., \& Dettmar, R.-J. 2009, A\&A, 504, 807

Stadel, J., Potter, D., Moore, B., Diemand, J., Madau, P., Zemp, M., Kuhlen, M., \& Quilis, V. 2009, MNRAS, 398, L21

Stark, D.V., McGaugh, S.S., \& Swaters, R.A. 2009, AJ, 138, 392 (S09)

Swaters, R.A., Sanders, R.H., \& McGaugh, S.S. 2010, ApJ, 718, 380 (S10)

Trachternach, C., de Blok, W.J.G., Walter, F., Brinks, E., \& Kennicutt, R.C. 2008, AJ, 136, 2720

van den Hoek, L.B., de Blok, W.J.G., van der Hulst, J.M., \& de Jong, T. 2000, A\&A, 357, 397 (vdH00)

van Zee, L. 2000, AJ, 119, 2757 (vZ00)

van Zee, L. 2001, AJ, 121, 2003 (vZ01)

Vorobyov, E.I., Shchekinov, Yu., Bizyaev, D., Bomans, D., \& Dettmar, R.-J. 2009, A\&A, 505, 483

Weinberg, M.D., \& Katz, N. 2002, ApJ, 580, 627

Wyder, T.K. et al. 2009, ApJ, 696, 1834 (W09)

Zwaan, M.A., van der Hulst, J.M., de Blok, W.J.G., \& McGaugh, S.S. 1995, MNRAS, 273, L35 
TABLE 1

Late-type LSB Galaxy Properties

\begin{tabular}{|c|c|c|c|c|c|c|c|c|c|c|c|}
\hline $\begin{array}{c}\text { Galaxy } \\
(1)\end{array}$ & $\begin{array}{c}V_{\text {flat }} \\
\left(\mathrm{km} \mathrm{s}^{-1}\right) \\
(2)\end{array}$ & $(3)$ & $\begin{array}{c}\Delta_{V / 2} \\
\left(10^{5}\right) \\
(4)\end{array}$ & $\begin{array}{c}M_{t o t a l} \\
\left(10^{10} M_{\odot}\right) \\
(5)\end{array}$ & $\begin{array}{c}M_{H I} \\
\left(10^{10} M_{\odot}\right) \\
(6)\end{array}$ & $\begin{array}{c}(B-V) \\
(\operatorname{mag}) \\
(7)\end{array}$ & $\begin{array}{c}M_{\star} \\
\left(10^{10} M_{\odot}\right) \\
(8)\end{array}$ & $\begin{array}{c}12+\log (\mathrm{O} / \mathrm{H}) \\
(9)\end{array}$ & $\begin{array}{c}\mathrm{SFR} \\
M_{\odot \mathrm{yr}^{-1}} \\
(10)\end{array}$ & $\begin{array}{c}<\mathrm{SFR}>_{\text {past }} \\
M_{\odot \mathrm{yr}^{-1}} \\
(11)\end{array}$ & $\begin{array}{c}\text { References } \\
\text { (12) }\end{array}$ \\
\hline F568-1 & 142 & 0.41 & 4.5 & 5.7 & 0.50 & 0.58 & 0.31 & 8.62 & $0.074^{\mathrm{a}}, 0.28^{\mathrm{b}}$ & $0.52^{\mathrm{a}}$ & D01;K07;S09;vdH00;W09 \\
\hline F568-3 & 120 & 0.48 & 1.8 & 5.6 & 0.39 & 0.61 & 0.41 & 8.68 & $0.35^{\mathrm{b}}$ & $\ldots$ & K08;M05;S09;vdH00;W09 \\
\hline F568-V1 & 118 & 0.26 & 4.3 & 5.6 & 0.34 & 0.57 & 0.24 & 8.61 & $0.29^{\mathrm{b}}$ & $0.135^{\mathrm{a}}$ & D01;K07;S09;vdH00;W09 \\
\hline F563-V2 & 113 & 0.45 & 3.9 & 2.6 & 0.32 & 0.51 & 0.25 & 8.10 & $0.18^{\mathrm{a}}$ & $0.13^{\mathrm{a}}$ & $\mathrm{H} 05 ; \mathrm{K} 07 ; \mathrm{K} 04 ; \mathrm{K} 08 ; \mathrm{S} 09$ \\
\hline F579-V1 & 114 & 0.46 & 20 & 4.3 & 0.11 & 0.70 & 0.53 & $\cdots$ & $0.27^{\mathrm{b}}$ & $\ldots$ & B00;D01;D96;S09;W09 \\
\hline F563-1 & 111 & 0.38 & 3.1 & 5.2 & 0.39 & 0.64 & 0.20 & 8.02 & $0.17^{\mathrm{b}}$ & $\ldots$ & K04;K08;S09;W09 \\
\hline F574-1 & 100 & 0.43 & 3.1 & 2.9 & 0.49 & 0.49 & 0.29 & & $0.33^{\mathrm{b}}$ & $\ldots$ & B00;D01;S09;W09 \\
\hline UGC 5005 & 99 & 0.50 & 0.67 & 6.2 & 0.41 & $\ldots$ & $\ldots$ & 8.04 & $\ldots$ & $\ldots$ & D02;D98;K04 \\
\hline F583-1 & 86 & 0.30 & 1.8 & 2.5 & 0.16 & 0.39 & 0.025 & & $0.055^{\mathrm{a}}, 0.087^{\mathrm{b}}$ & $\ldots$ & H05;K08;S09;W09 \\
\hline UGC 3371 & 86 & 0.38 & 1.2 & 1.8 & 0.12 & 0.72 & 0.18 & 8.48 & $0.092^{\mathrm{a}}$ & $0.233^{\mathrm{a}}$ & D02;H99;S09;vZ00;vZ01 \\
\hline NGC 4395 & 83 & 0.47 & 12 & 1.3 & 0.20 & $\ldots$ & $\ldots$ & 8.27 & $\ldots$ & $\ldots$ & K08;P04 \\
\hline UGC 5750 & 79 & 0.45 & 0.39 & 3.1 & 0.14 & $\ldots$ & $\ldots$ & $\ldots$ & $0.27^{\mathrm{b}}$ & $\ldots$ & D98;K08;W09 \\
\hline NGC 3274 & 79 & 0.49 & 39 & 1.0 & 0.085 & $\ldots$ & 0.10 & 8.33 & $\ldots$ & $\cdots$ & D02;H99;S09 \\
\hline NGC 1560 & 78 & 0.57 & 6.0 & 1.2 & 0.12 & 0.57 & 0.050 & 8.02 & $\ldots$ & $\ldots$ & $\mathrm{D} 02 ; \mathrm{P} 04 ; \mathrm{S} 09$ \\
\hline UGC 731 & 75 & 0.36 & 15 & 0.91 & 0.074 & 0.34 & 0.18 & 8.47 & $0.16^{\mathrm{a}}$ & $0.041^{\mathrm{a}}$ & D02;H99;K07;S09;S10 \\
\hline F583-4 & 70 & 0.38 & 4.9 & 0.76 & 0.077 & & $\ldots$ & $\ldots$ & $\ldots$ & $\ldots$ & D98;K08 \\
\hline NGC 4455 & 64 & 0.62 & 2.4 & 0.56 & 0.061 & 0.10 & $\ldots$ & $\ldots$ & $0.37^{\mathrm{a}}$ & $6.61^{\mathrm{a}}$ & $\mathrm{D} 02 ; \mathrm{K} 07 ; \mathrm{S} 10$ \\
\hline UGC 4173 & 57 & 0.62 & 0.41 & 0.91 & 0.24 & $\ldots$ & $\ldots$ & $\ldots$ & $\ldots$ & $\ldots$ & $\mathrm{D} 02 ; \mathrm{S} 10$ \\
\hline NGC 2366 & 55 & 0.62 & 4.7 & 0.38 & 0.093 & $\ldots$ & 0.13 & 7.92 & $0.095^{\mathrm{a}}$ & $\ldots$ & $\mathrm{D} 02 ; \mathrm{H} 05 ; \mathrm{P} 04 ; \mathrm{S} 09$ \\
\hline DDO 185 & 50 & 0.34 & 1.1 & 0.13 & 0.013 & 0.43 & $\ldots$ & 7.70 & $0.14^{\mathrm{a}}$ & $0.009^{\mathrm{a}}$ & D02;K07;L07 \\
\hline
\end{tabular}

odels; see references. Col. (4): $\Delta_{V / 2}$ parameter of Alam et al. (2002) Col. (5). Total dynamical mass calculated at the last rotation curve point Col. (6): Total H mass. Col. (7): Optical color. Col. (8): Stellar mass calculated using the $\Upsilon_{\star}-(B-V)$ relation of Stark et al. (2009). Col. (9): Mean oxygen abundance. Col. (10):

Current star formation rate. Col. (11): Average past star formation rate.

$\mathrm{H} \alpha$

${ }^{\mathrm{b}} U V$ 